\title{
Why do we need yet another journal?
}

\author{
Valentin Fuster
}

Welcome to Nature Clinical Practice Cardiovascular Medicine, one of a new series of clinical journals from the publishers of Nature.

I have chosen to work with Nature Clinical Practice Cardiovascular Medicine first and foremost because of Nature Publishing Group's well-established reputation for excellence. I also believe that in the crowded market place of specialty journals, a role remains for high-quality, relevant information in a convenient format.

Nature Clinical Practice Cardiovascular Medicine believes that, with the enormous amount of original research published, it is impossible for busy practicing doctors, specialists and subspecialists to keep abreast of all the developments in their field. Therefore, our aim is to provide an authoritative overview of key developments in an easy-to-read, stimulating format to expand the knowledge base and impact on the way clinicians treat their patients.

This journal has several unique editorial features that we hope will ease your reading workload and help you to interpret research at a practical level. Nature Clinical Practice Cardiovascular Medicine effectively reads for you, acting as a filter for publications in the highest-impact cardiovascular and general medical journals, as well as highly relevant papers in journals that publish original research in the specialty less frequently.

Several types of article will be presented every month. The Editorial is a topical onepage article, and will in future issues be written by me or a nominee. Second, the Viewpoints present the opinions of leading specialists, again discussing topical issues in cardiovascular medicine. Third, Research Highlights summarize original and evidence-based articles published elsewhere. Practice Point articles focus on the highest-quality original research

\section{In the crowded market place of specialty journals, a role remains for \\ high-quality, relevant information in a convenient format}

$V$ Fuster is the Editorin-Chief of Nature Clinical Practice Cardiovascular Medicine, Director of the Zena and Michael A Wiener Cardiovascular Institute and the Marie-José and Henry R Kravis Center for Cardiovascular Health, and the Richard Gorlin, MD/Heart Research Foundation Professor.

Competing interests The author declared he has no competing interests.

www.nature.com/clinicalpractice doi:10.1038/ncpcardio0026 reports. Each consists of a structured synopsis written by the Nature Clinical Practice editorial team, accompanied by a commissioned commentary written by a leading authority in the field to put the original article into context. A one-sentence Practice Point underscores how the findings should affect the way clinicians treat their patients. These articles will appear in the first half of the journal.

The second half of the journal consists of timely and authoritative commissioned reviews and case studies. As well as general reviews, there are several specialized review types that will appear, various combinations of which will appear in each month's issue. Therapy Insights explore medical conditions treated in other specialties that might have cardiovascular manifestations, and what cardiovascular physicians should know for effective management of patients (e.g. Marfan's syndrome, as discussed in this issue). Drug Insights and Technology Insights review the important emerging and established information doctors need to know about drug classes, technologies or techniques. Mechanisms of Disease articles present the scientific background of disease development. Primer articles explain clearly how to interpret trials and discuss good practice. Finally, Case Studies will be several pages long and will cover uncommon, and not so uncommon, presentations, for which the discussion will have an educational emphasis.

To ensure the highest quality in the journal, its international advisory board will aid good coverage of all fields. In addition, all content, except the Editorial and the Research Highlights, will undergo peer-review.

Given the variety of content, I truly believe Nature Clinical Practice Cardiovascular Medicine will be an invaluable tool to academic and practicing doctors, and it promises to become a premiere cardiovascular journal. 